\title{
Dirk Vanderbeke \\ Überlegungen zur Ästhetik in der Literatur und in den Naturwissenschaften
}

\begin{abstract}
Seit den Anfängen der neueren Wissenschaftsgeschichte im siebzehnten Jahrhundert und der damit einhergehenden Forderung nach einer einfachen und neutralen Wissenschaftssprache wird die ästhetische Wahrnehmung traditionell der Literatur und der Kunst zugeordnet, während die Wissenschaft nicht nach Schönheit, sondern nach Wahrheit strebt. Diese einfache Trennung wird allerdings von Vertretern beider Bereiche infrage gestellt. So wie es in der Literatur auch immer um die Suche nach einer Wahrheit ging, so finden sich auch in der wissenschaftlichen Arbeit Momente und Phänomene, Theorien und mathematische Gleichungen, die ein ästhetisches Empfinden hervorrufen und als schön wahrgenommen werden. Diese Erfahrungen und ihre Bedingungen sollen im Folgenden untersucht und dabei mit ästhetischen Konzepten der Literaturwissenschaft in Verbindung gesetzt werden.
\end{abstract}

Wenn wir über Ästhetik in der Wissenschaft sprechen, dann sind dabei meines Erachtens zwei grundsätzlich unterschiedliche Phänomene angesprochen, die Schönheit, die wir vorfinden, und die Schönheit, die in unserem Verstehen und unseren theoretischen Zugängen zu der Natur zum Ausdruck kommt. In seinem Vortrag über „Die Bedeutung des Schönen in der exakten Naturwissenschaft“ nimmt Werner Heisenberg eine ähnliche Unterscheidung vor, die er schon in der Antike angelegt sieht:

Die eine bezeichnet die Schönheit als die richtige Übereinstimmung der Teile miteinander und mit dem Ganzen. Die andere, auf Plotin zurückgehend, ohne jede Bezugnahme auf die Teile, bezeichnet sie als das Durchleuchten des ewigen Glanzes des „Einen“ durch die materielle Erscheinung. ${ }^{1}$

Analog dazu hat Freeman Dyson in einem Artikel in Science die beiden wissenschaftsgeschichtlichen Ansätze von Thomas S. Kuhn und Peter Galison erörtert. Danach ging es bei Galison um die Geschichte der wissenschaftlichen Werkzeuge, mit denen wir die Natur erschließen können - paradigmatisch dafür stehen das Teleskop und das Mikroskop -, wobei der Übergang von analogen zu digitalen Instrumenten im Vordergrund steht. Kuhn dagegen legte den Schwerpunkt auf

1 Werner Heisenberg: Die Bedeutung des Schönen in den exakten Naturwissenschaften. In: ders.: Schritte über Grenzen. Erw. Neuausg. München: Piper, 1977. S. 289. 
die Bedeutung von Ideen - so hätten Einstein, Heisenberg, Schrödinger und Dirac „die Geheimnisse der Natur erraten, weil sie Träume von mathematischer Schönheit träumten“. ${ }^{2}$ Es geht also, wenn wir die beiden historischen Ansätze unter dem Gesichtspunkt der Ästhetik betrachten, zum einen um die Schönheit der Natur, wie sie sich in der Betrachtung zeigt - auch in der Betrachtung mit technischen Hilfsmitteln -, und zum anderen um die Schönheit der Theorien und das daraus folgende Durchdringen und Verstehen.

Unsere Vorstellungen von der Schönheit der Natur folgen in mancher Hinsicht den Konzepten des Naturschönen, wie sie in der Romantik entwickelt wurden und inzwischen auch auf unterschiedliche Weise wissenschaftlich erfasst wurden. Dies geschieht einerseits in den Forschungen zur neuronalen Ästhetik, andererseits in Überlegungen zu einer evolutionären Ästhetik, ${ }^{3}$ wobei diese beiden Forschungszweige durchaus miteinander verbunden werden können. Schönheit in der Natur findet sich u. a. in den Kunstformen der Natur, wie sie von Ernst Haeckel eindrucksvoll vorgeführt wurden, in den vielfältigen spektakulären, aber auch technisch aufbereiteten Hubble-Bildern oder auch in den Fotos des Monats, die in jeder Ausgabe von Spektrum der Wissenschaft enthalten sind.

Wichtiger für diesen Beitrag ist die Schönheit der Theorien. Bei dem Versuch, die Ästhetiken in den Wissenschaften und in der Literatur in eine Verbindung $\mathrm{zu}$ bringen und auch voneinander abzugrenzen, geht es weniger um das, was wir vorfinden, als um die Zugänge, die wir wählen, um uns mit der Wirklichkeit, wie sie von uns wahrgenommen wird, auseinanderzusetzen. Die ästhetischen Momente, die hier zum Ausdruck kommen, sind von Menschen geschaffen, und stehen daher in einer Zwischenposition; einerseits müssen sie den externen Gegebenheiten genügen und andererseits sind sie interne Konstruktionen, die den Regeln unseres Wahrnehmungs- und ,Denkapparates' folgen. Genauere Beurteilungen dieser Zwischenposition können sehr weit voneinander abweichen und reichen von den Annahmen eines starken Realismus, nach dem die Welt so ist, wie wir sie wahrnehmen, bis zu einer evolutionären Erkenntnistheorie und zu unterschiedlich radikalen relativistischen und konstruktivistischen Konzepten. Eine weitere Möglichkeit, sich dem Thema der Ästhetik in Wissenschaft und Kunst bzw. Literatur zu nähern, besteht in der Annahme einer gegenseitigen Befruchtung, unter Umständen mit einem Rekurs auf den jeweils vorherrschen-

2 Freeman Dyson: Is Science Mostly Driven by Ideas or by Tools? In: Science 338 (14. Dezember 2012). S. 1426-1427. Dt. Übers.: Theorie und Werkzeug. In: Süddeutsche Zeitung. 14. Dezember 2012. S. 18.

3 Vgl. z. B. Olaf Breidbach: Neuronale Ästhetik. München: Wilhelm Fink, 2013. S. 83-94; Dennis Dutton: The Art Instinct. Beauty, Pleasure, and Human Evolution. New York u. a.: Bloomsbury Press, 2010. S.13-28. 
den Zeitgeist. Selbstverständlich gehört die Wissenschaft zu den Bereichen, mit denen sich Kunst und Literatur kontinuierlich auseinandersetzen, und damit sind Ästhetisierungen wissenschaftlicher Erkenntnisse oder auch die metaphorische Verarbeitung wissenschaftlicher Konzepte ein Bestandteil vieler Texte und Kunstwerke - die Bestätigung für die Annahme, wissenschaftliche Theorien hätten Eingang in literarische Texte gefunden, lässt sich gewöhnlich durch einen Blick in die sogenannten acknowledgements oder Danksagungen der Autoren bestätigen. Der umgekehrte Weg lässt sich nicht ganz so einfach gehen, und es gibt sicher nicht viele Wissenschaftler, die bereitwillig erklären würden, sie wären in ihrer Arbeit von einem Roman, Gedicht oder Gemälde inspiriert worden. Dabei werden von literaturwissenschaftlicher Seite gelegentlich komplexe Feedbackmechanismen angenommen, durch die die Kunst und Literatur einer Epoche dann doch eine Wirkung auf die Wissenschaftler und ihr Denken gehabt haben könnten, sodass Fälle, in denen Analogien zwischen Kunstwerken und späteren wissenschaftlichen Entdeckungen oder Theorien bestehen oder konstruiert werden können, als Beispiele für eine Antizipation und Einflussnahme verstanden werden. ${ }^{4}$

Hier kann allerdings auch eine Analogie zur Selektion in evolutionären $\mathrm{Zu}$ sammenhängen herangezogen werden, um allzu einfache relativistische Interpretationen derartiger Ähnlichkeiten infrage zu stellen. ${ }^{5}$ Laut Darwin gibt es zwei wesentliche Selektionsmechanismen: die natürliche Selektion und die sexuelle Selektion. Die natürliche Selektion bewirkt, dass diejenigen Organismen, die an spezifische Lebensumstände oder -räume besser angepasst sind, überleben, während weniger gut angepasste Lebensformen über kurz oder lang verschwinden werden. Die sexuelle Selektion dagegen führt dazu, dass bestimmte Individuen einer Spezies aufgrund spezifischer Eigenschaften in der Konkurrenz um Fortpflanzungsmöglichkeiten bevorzugt werden und daher ihre Eigenschaften einer größeren Anzahl von Nachkommen weitervererben. Die Kriterien für diese Auswahl können eingängig sein - z. B. Größe oder Stärke und daraus resultierend bessere Befähigungen beim Nahrungserwerb -, aber auch kontraintuitiv wie der überlange Schwanz des Paradiesvogels. Die entsprechenden Signale und ihre Wahr-

4 Vgl. z. B. N. Katherine Hayles: Introduction. Complex Dynamics in Literature and Science. In: Chaos and Order. Complex Dynamics in Literature and Science. Hrsg. von N. K. Hayles. Chicago: University of Chicago Press, 1991. S. 1-33, hier S. 7; oder Thomas P. Weissert: Representation and Bifurcation. Borges's Garden of Chaos Dynamics. In: Chaos and Order. Complex Dynamics in Literature and Science. Hrsg. von N. K. Hayles. Chicago: University of Chicago Press, 1991. S. 223-243, hier S. 224.

5 Vgl. zum Folgenden auch Dirk Vanderbeke: Theoretische Welten und literarische Transformationen. Tübingen: Niemeyer, 2004. S. 133-172. Dort werden die möglichen Analogien zwischen der Evolution und der Entwicklung wissenschaftlicher Theorien wesentlich ausführlicher behandelt. 
nehmung können als eine Analogie zu ästhetischem Empfinden verstanden werden, d. h. das schönere Exemplar wird bei der Partnerwahl vorgezogen.

In Analogie dazu müssen wissenschaftliche Theorien ebenfalls zwei Selektionsmechanismen durchlaufen. Zum einen müssen sie überhaupt wahrgenommen werden, d. h. die Arbeit, in der sie vorgestellt werden, muss veröffentlicht werden. Dazu müssen sie bestimmten formalen Kriterien genügen - wobei ästhetische Aspekte durchaus eine Rolle spielen können - und dann auch noch innerhalb der wissenschaftlichen Gemeinschaft aufgenommen werden, was selbstverständlich immer nur als eine vorläufige Akzeptanz verstanden werden kann. Wenn dies geschehen ist, folgt jedoch die zweite Selektion, denn die neue Theorie muss auch noch in der Natur überleben und sich gegenüber konkurrierenden Konzepten durchsetzen können. Und hier sollte die Ästhetik eigentlich keine wesentliche Rolle mehr spielen, es sei denn, die Wahrnehmung der Wissenschaftler wäre so an der Natur geschult, dass mit einer feststellbaren Regelmäßigkeit zutreffende Theorien auch als schöner wahrgenommen werden.

Ebendiese Möglichkeit nimmt Steven Weinberg an. Sein Buch Dreams of a Final Theory enthält ein Kapitel mit dem Titel „Beautiful Theories“. Darin führt Weinberg aus, dass sich schöne wissenschaftliche Theorien selbstverständlich von schönen Bildern und Gedichten unterscheiden und dass ein Gefühl für die Schönheit einer Theorie einem Zweck dient: „[I]t is supposed to help the physicist select ideas that help us to explain nature““. ${ }^{6}$ Er schreibt an anderer Stelle:

[I]n this century, as we have seen in the cases of general relativity and the electroweak theory, the consensus in favour of physical theories has often been reached on the basis of aesthetic judgement before the experimental evidence for these theories became really compelling. I see in this the remarkable power of the physicist's sense of beauty acting in conjunction with and sometimes even in opposition to the weight of experimental evidence. ${ }^{7}$

Später spricht er dann noch einmal direkt den evolutionären Prozess an, der hier wirksam gewesen sein könnte:

[T] he universe itself acts on us as a random, inefficient, and yet in the long run effective, teaching machine. Just as through an infinite series of accidental events, atoms of carbon and nitrogen and oxygen and hydrogen joined together to form primitive forms of life that later evolved into protozoa and fishes and people, in the same manner our way of looking at the universe has gradually evolved through a natural selection of ideas. Through countless false starts, we have gotten it beaten into us that nature is a certain way, and we have grown to look at that way that nature is as beautiful. [...] One of the things that make the history

6 Steven Weinberg: Dreams of a Final Theory. London: Vintage, 1993. S. 106.

7 Ebd., S. 102-103. 
of science so endlessly fascinating is to follow the slow education of our species in the sort of beauty to expect in nature. ${ }^{8}$

Dies läuft letztlich auf eine Annahme hinaus, die wohl am besten von John Keats in seinem Gedicht „Ode on a Grecian Urn“ formuliert wurde:

Beauty is truth, truth beauty, - that is all

Ye know on earth, and all ye need to know. ${ }^{9}$

Diese Argumentation ist problematisch, denn sie verschiebt die ästhetische Wahrnehmung von der meist angenommenen Hinwendung zu schönen - d.h. dem Überleben und der Fortpflanzung dienlichen - Umgebungen und Partnern zu abstrakten Problemlösungsstrategien, die in der Lebenswelt unserer prähistorischen Vorfahren kaum eine Rolle gespielt haben dürften. Roger Penrose schreibt dementsprechend etwas relativierend: „For our remote ancestors, a specific ability to do sophisticated mathematics can hardly have been a selective advantage, but a general ability to understand could well have.“"10

Auf dem derzeitigen Büchermarkt, wird Penroses Vorbehalt allerdings nicht immer beachtet. So hat der Mathematiker Keith Devlin ein Buch mit dem Titel The Maths (bzw. Math) Gene veröffentlicht - der Untertitel lautet, je nachdem welche Ausgabe man kauft: Why everyone has it, but most people don't use it bzw. How Mathematical Thinking Evolved and Why Numbers Are Like Gossip. Auch Devlin nimmt allerdings schon gleich im Vorwort die eher starke Behauptung, die der Titel impliziert, wieder zurück, wenn er schreibt:

Before we begin, I should clear up one thing: there is no ,maths gene' in the sense of a specific sequence of human DNA that confers mathematical ability. There are, of course, genes that affect our ability to do mathematics. But, in calling this book „The Maths Gene“, I am simply adopting a common metaphor. ${ }^{11}$

Er geht also von einer grundsätzlichen menschlichen Befähigung zur Mathematik aus, die uns angeboren ist, aber nicht von einer lokalisierbaren Gensequenz von Seiten der Evolutionsbiologie könnte man hier wohl eher von epigenetischen Regeln sprechen, die mathematisches Denken begünstigen.

8 Ebd., S. 125-126.

9 John Keats: Ode on a Grecian Urn. In: Romantic Poetry. An Annotated Anthology. Hrsg. von Michael O’Neill und Charles Mahoney. Malden, MA: Blackwell, 2008. S. 451.

10 Roger Penrose (mit Abner Shimony, Nancy Cartwright und Stephen Hawking): The Large, the Small, and the Human Mind. Cambridge: Cambridge UP, 1999. S. 114.

11 Keith Devlin: The Maths Gene. Why everyone has it but most people don't use it. London: Phoenix, 2001. S. 4. 
In seinem Buch geht Devlin allerdings auch auf angenommene Parallelen zwischen mathematischer und künstlerischer/literarischer Imagination aus, und diese Überlegungen wurden dann auch mit Interesse bei Geisteswissenschaftlern aufgenommen, die sich mit der Verbindung von Naturwissenschaft und Literatur beschäftigen. So erschien im Winter 2009 eine Nummer der Zeitschrift Configurations. A Journal of Literature, Science and Technology zu dem Thema „Mathematics and Imagination“, in der bereits auf der ersten Seite der Einleitung unter Verweis auf Devlins Buch eine Verbindung zwischen Mathematik und Literatur gezogen wird:

„Is there a link between doing mathematics and reading a novel“, Devlin asks. „Very probably“, he answers. Imagining a conversation between two invented characters or the intricate imagery of a poem arguably requires a similar mental process as imagining „the square root of minus fifteen“, as mathematician Barry Mazur has demonstrated. ${ }^{12}$

Der Gedanke, dass eine der einfachsten und üblichsten Imaginationsleistungen mit einer der komplizierteren und zunächst einmal kontraintuitiven mathematischen Konstruktionen gleichzusetzen ist, dürfte wohl nicht nur gewöhnliche Leser und Leserinnen erstaunen, sondern auch Mathematiker. Bei Devlin selbst hört sich das etwas weniger radikal an, wenn er schreibt:

To the ordinary person the number $\pi$ is just that: a number - what you get when you divide the circumference of a circle by its diameter. [...] But to me, $\pi$ has a definite personality. It is a major character in a drama that has been unfolding on a vast landscape for the past 2500 years. [...] Whenever I see a reference to $\pi$, I at once have a mental image of it, along with all its connections, just as a mention of a hero in a novel will bring to mind a rich picture of that individual, complete with all his relationships. ${ }^{13}$

Es dürfte zumindest für Geisteswissenschaftler beruhigend sein, dass normale Menschen eine Zahl wie $\pi$ noch einfach als Zahl wahrnehmen können und es sich bei der Analogie mit fiktionalen Texten doch eher um eine recht eigenwillige und persönliche Sicht handelt. Die Verbindung zwischen mathematischer und literarischer Imagination wird dann noch einmal deutlich relativiert, wenn Devlin schreibt, dass Romanautoren ihrer Phantasie verhältnismäßig freien Lauf lassen können, während mathematische Imagination klaren Einschränkungen unterliegt. Und auch der Mathematiker Barry Mazur erklärt gleich zu Beginn seines Buches Imagining Numbers (Particularly the Square Root of Minus Fifteen),

12 Arielle Saibler und Henry S. Turner: Mathematics and the Imagination. A Brief Introduction. In: Configurations. A Journal of Literature, Science and Technology 17.1-2 (Winter 2009). S.1-18, hier S. 1.

13 Devlin: The Maths Gene, S. 246. 
ganz im Gegensatz zu der Sicht, die Arielle Saiber und Henry S. Turner ihm zuschreiben, „Of course, mathematics and poetry are far apart“. ${ }^{14}$ Sein Interesse liegt nicht darin, die mentalen Prozesse gleichzusetzen, sondern eher einer interessierten Leserschaft, die nicht umfassend mathematisch vorgebildet ist, $\mathrm{zu}$ demonstrieren, dass auch hier imaginative Zugänge möglich sind.

Die Frage, welche Rolle die Imagination bei der wissenschaftlichen Arbeit spielen kann, berührt auch Aspekte der organisierten Wissensproduktion. Klaus Mecke trifft in seinem Beitrag zu diesem Band unter Berufung auf Thomas Lehr die Unterscheidung zwischen Ereignis und Erfahrung - das Ereignis ist eine einmalige und nicht-wiederholbare Wahrnehmung, während die Erfahrung persistent, mitteilbar und prägend ist. ${ }^{15}$ In ähnlicher Weise lässt sich ein Unterschied zwischen dem Moment und dem Inhalt von Erkenntnis formulieren, wobei der Moment - nennen wir ihn die Entdeckung - ebenso einmalig und unwiederholbar ist wie das Erlebnis, während der Inhalt - also das Wissen - dauerhaft und vermittelbar ist. Der Moment der Entdeckung lässt sich allerdings nicht immer klaren und festlegbaren Regeln unterwerfen. William Whewell schrieb schon 1840:

But in truth, we must acknowledge, before we proceed with this subject, that, speaking with strictness, an Art of Discovery is not possible; - that we can give no Rules for the pursuit of truth which shall be universally and peremptorily applicable; - and that the helps which we can offer to the inquirer in such cases are limited and precarious. ${ }^{16}$

Dabei ist es kaum möglich, den Moment der Erkenntnis greifbar zu machen. Er ist nicht planbar oder vorhersehbar, und, wie die Autobiographien von Wissenschaftlern es gelegentlich beschreiben, ist er auch nicht notwendigerweise an den Arbeitsplatz gebunden. Auch wenn der Fall des Apfels, der Newton zu seiner Erkenntnis gebracht haben soll, vermutlich nicht viel mehr ist als eine Legende, so gibt es doch genug Erinnerungen und Berichte, nach denen eine wissenschaftliche Erleuchtung quasi spontan oder unter ungewöhnlichen Umständen zustande kam. So berichtete Hermann Weyl, Erwin Schrödinger „did his great work during a late erotic outburst in his life“, ${ }^{17}$ konkret: als er Weihnachten

14 Barry Mazur: Imagining Numbers (Particularly the Square Root of Minus Fifteen). London: Penguin, 2004. S. XV.

15 Vgl. den Beitrag von Klaus Mecke in diesem Band (Zahl und Erzählung), S. 31-83, hier S. 33.

16 William Whewell: The Philosophy of the Inductive Sciences. Founded Upon Their History. Bd. 2. London: John W. Parker, 1840. S. 483. Vgl. dazu auch Peter Medewar, der ebendies in seinem Essay „Is the Scientific Paper Fraudulent?“ (The Saturday Review. 1. August 1964. S. 42-43) zitiert, um die Vorstellung von der wissenschaftlichen Arbeit als einem fein säuberlich organisierten, induktiven Prozess zu unterlaufen.

17 Zit. nach Walter J. Moore: A Life of Erwin Schrödinger. Cambridge: Cambridge UP, 1994. S. 138. 
1925 mit einer Geliebten, deren Identität ungeklärt ist, einen Skiurlaub in Arosa verbrachte. „Like the dark lady who inspired Shakespeare's Sonnets, the lady of Arosa may remain forever mysterious." ${ }^{18}$ Andrew Wiles beschrieb den Moment der Erkenntnis, in dem er den Beweis der Fermatschen Vermutung endlich durchdrang, als eine Erleuchtung und gleichzeitig als ästhetische Erfahrung: „Suddenly, totally unexpectedly, I had this incredible revelation. [...] It was so indescribably beautiful, it was so simple and so elegant, and I just stared in disbelief for twenty minutes“. ${ }^{19}$

Auf ähnliche Weise schreibt Werner Heisenberg über den Moment, in dem es ihm sehr spät in einer Nacht gelungen war, eine geschlossene und widerspruchsfreie mathematische Lösung für die Verbindung des Energieerhaltungssatzes mit der Quantenmechanik zu finden:

\begin{abstract}
Im ersten Augenblick war ich zutiefst erschrocken. Ich hatte das Gefühl, durch die Oberfläche der atomaren Erscheinung hindurch auf einen tief darunter liegenden Grund von merkwürdiger innerer Schönheit zu schauen, und es wurde mir fast schwindlig bei dem Gedanken, daß ich nun dieser Fülle von mathematischen Strukturen nachgehen sollte, die die Natur vor mir ausgebreitet hatte. ${ }^{20}$
\end{abstract}

Hier lässt sich sehr gut der Aspekt erkennen, den er auch in seinen Überlegungen über „Die Bedeutung des Schönen in den exakten Naturwissenschaften“ ausgeführt hatte, eine Art säkularer mystischer Erleuchtung, die den Verstehensoder Durchdringungsmoment auszeichnet. Die Erinnerung weist ganz deutlich Elemente des Erhabenen auf, ${ }^{21}$ eine Verbindung von Schrecken und Erschauern, oder, wie es Edmund Burke nannte, eine Art „delightful horror“.22

Heisenbergs Erinnerung verweist dabei aber auch auf einen anderen Text, und ich möchte diese vielleicht nicht ganz zufällige Verbindung dazu benutzen, auf einige Aspekte der Ästhetik in Wissenschaft und Literatur näher einzugehen. Es handelt sich um einen Text, in dem der Erzähler ebenfalls erschauernd und überwältigt in die Tiefe schaut, er erblickt darin jedoch nicht das strahlende Licht

18 Ebd., S.141.

19 Zit. nach Charles J. Mozzochi: The Fermat Diary. Providence, RI: American Mathematical Society, 2000. S. 55-56.

20 Werner Heisenberg: Der Teil und das Ganze [1969]. München: dtv, 1987. S. 78.

21 Vgl. Wolfgang Braungart und Silke Jakobs: Staunen und Hingabe. Zur Ästhetik des Wissens seit dem 18. Jahrhundert. In: Ästhetik der Wissenschaft. Interdisziplinärer Diskurs über das Gestalten und Darstellen von Wissen [Zeitschrift für Ästhetik und Allgemeine Kunstwissenschaft. Sonderheft 7]. Hrsg. von Wolfgang Krohn. Hamburg: Meiner, 2006. S. 201-218, hier S. 211.

22 Edmund Burke: A Philosophical Enquiry into the Origin of our Ideas of the Sublime and Beautiful [1757]. Hrsg. von Adam Phillips. Oxford u. a.: Oxford UP, 1990, S. 67. 
einer wissenschaftlichen Wahrheit, sondern das gleichfalls strahlende göttliche Licht:
O Gnadenmeer, das mich mit Mut bewehrte, zu blicken tief ins Licht, ins ewigreine, Bis meine Sehkraft sich darin verzehrte! In seiner Tiefe sah ich im Vereine, $\mathrm{Zu}$ einem Band gebunden durch die Liebe, Was sonst im Weltenbuch zerstreut erscheine: Wesen, Zufälligkeit und ihr Getriebe Gleichsam verschmolzen in so fester Bindung, Daß was ich spräche, blasser Schein nur bliebe. ${ }^{23}$

Die Schau ist damit noch nicht abgeschlossen, und Dante führt eine weitere Metapher ein, um die göttliche Natur und seine Sicht, die alle Worte übersteigt, zum Ausdruck zu bringen.
Ich drang zum tiefen klaren Licht als Ganzes, Und sah gleich groß drei Kreise hell gezogen. Doch anders war die Farbe jedes Kranzes.
Wie Iris Iris, spiegelte ein Bogen
Den anderen. Und der Dritte, überschwänglich
An Glut, schien aus den zweien gleichstark zu wogen. Oh wie ist schwach mein Wort und wie verfänglich
Für mein Verstehn! ${ }^{24}$

Die erste Interpretation dieser Textpassage ist relativ einfach: Die drei Kreise stellen die Dreifaltigkeit dar. An dieser Stelle möchte ich noch einmal auf Klaus Meckes Beitrag in diesem Band zurückkommen. ${ }^{25}$ Er diskutiert darin u. a. die heuristischen Potentiale der Metapher und die Funktion von Metaphern im wissenschaftlichen Diskurs, darunter beispielsweise die Metapher „grüne Quarks“. So gibt es das Wort ,quark' im Englischen erst seit 1939, als James Joyces Finnegans Wake erschien. Dort heißt es:

Three quarks for Muster Mark!

Sure he has not got much of a bark

And sure any he has it's all beside the mark..$^{26}$

23 Dante Alighieri: Die göttliche Komödie/Das neue Leben. Ital./dt. Übers. von Richard Zoozmann. Augsburg: Weltbild, 1994. „Paradies“, Canto 33, Z. 82-90. Herv. im Orig.

24 Ebd., Canto 33, Z. 115-122.

25 Vgl. den Beitrag von Klaus Mecke in diesem Band (Zahl und Erzählung), S. 31-83, hier S. 35-36 und $82-83$.

26 James Joyce: Finnegans Wake. London: Penguin, 1975. S. 383.1-3. 
Das ist nicht sehr luzide, und was genau ein Quark in Finnegans Wake ist, lässt sich nicht so richtig bestimmen. Es könnte sich um die Schreie von Seemöwen handeln, um Rufe im Allgemeinen, um das deutsche Wort ,Quark' oder auch um die drei Kinder der Hauptfigur, die manchmal in der Person von König Marke aus Tristram erscheint. Murray Gell-Mann übernahm das Wort, als er einen Begriff für die subatomaren Teilchen brauchte. Er schreibt dazu:

In 1963, when I assigned the name „quark“ to the fundamental constituents of the nucleon, I had the sound first, without the spelling, which could have been „kwork“. Then, in one of my occasional perusals of „Finnegans Wake“, by James Joyce, I came across the word „quark“ in the phrase „Three quarks for Muster Mark“. [...] From time to time, phrases occur in the book that are partially determined by calls for drinks at the bar. I argued, therefore, that perhaps one of the multiple sources of the cry „Three quarks for Muster Mark“ might be „Three quarts for Mister Mark“, in which case the pronunciation „kwork“ would not be totally unjustified. In any case, the number three fitted perfectly the way quarks occur in nature. ${ }^{27}$

Für die Physik ist die Sache damit abgeschlossen, der Begriff benötigt keine weitere Erklärung. Ein Quark ist das, was bestimmte Eigenschaften aufweist, wobei die Bestimmung dieser Eigenschaften sich durch neuere Entdeckungen ändern kann - der Begriff folgt dann schlicht der modifizierten Definition, er selbst hat keinen Einfluss darauf. Die eigentlich unzutreffende Beschreibung von Quarks durch Farben hat dann erneut eine metaphorische und gleichzeitig heuristische Komponente - durch die Verbindung der drei Farben entsteht die neutrale Farbe weiß, wie sich aus den unterschiedlichen Eigenschaften der Quarks die des Protons ergeben, das keine Farbwechselwirkung nach außen aufweist.

Die Metapher ist hier vollständig auflösbar, sie hat eine erklärende Funktion, die allerdings im Laufe der Zeit auch ihren Sinn verlieren und schließlich sogar

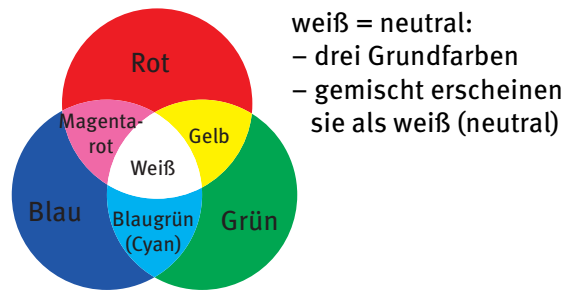

Abb. 1: Grafik: Klaus Mecke.

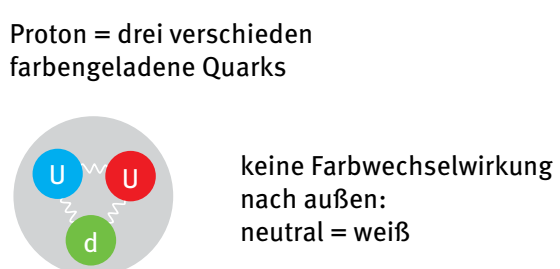

Abb. 2: Grafik: Klaus Mecke.

27 Murray Gell-Mann: The Quark and the Jaguar. New York: W. H. Freeman, 1994. S. 180-181. 
hinderlich werden kann. ${ }^{28}$ Stephen Jay Gould schreibt in diesem Zusammenhang, dass kanonische Bilder - und dazu gehören etablierte Metaphern in der Naturwissenschaft - auch eine einschränkende Wirkung haben können, indem sie Komplexitäten verdecken oder auch zu einer Gewöhnung führen, die dann das Denken in eingefahrenen Bahnen hält. Ein Beispiel für den ersten Fall wäre der Begriff des ,Spins‘ oder auch das Bohr'sche Atommodell, den zweiten Fall führt Gould genauer an einem Beispiel seines eigenen Faches aus, dem Begriff einer evolutionären Leiter. ${ }^{29}$

Insgesamt könnte man jedenfalls für die Naturwissenschaften von einer sich verengenden Bedeutung der Metapher sprechen, d.h. im Verlauf der Forschung stellt sich zunehmend heraus, inwieweit ein metaphorischer Begriff zutreffend ist und eine heuristische Funktion haben kann, und gegebenenfalls können Begriffe durch ihre Überprüfung ihre metaphorische Bedeutung weitgehend einbüßen oder auch aufgegeben werden. Man könnte in diesem Zusammenhang die Metapherntheorie von Max Black heranziehen, die selbst eine sehr anschauliche Metapher enthält. Black vergleicht die Metapher mit einem geschwärzten Glas, durch das wir den Nachthimmel betrachten:

Suppose I look at the night sky through a piece of heavily smoked glass on which certain lines have been left clear. Then I shall see only the stars that can be made to lie on the lines previously prepared upon the screen, and the stars I do see will be seen as organised by the screen's structure. ${ }^{30}$

Das organisierende Prinzip wäre hier die naturwissenschaftliche Forschung, durch die bestimmte Aspekte des Bildspenders zugelassen, andere jedoch geblockt werden. Im Umgang mit Literatur verhält es sich natürlich genau gegensätzlich. Man könnte hier, um bei dem Bild zu bleiben, von einem geradezu fanatischen Freikratzen neuer Linien sprechen, einer beständigen Suche nach neuen möglichen Bedeutungen einer literarischen Metapher, einer immer neuen Zuweisung neuer Sinnschichten im Kontext neuer theoretischer Modelle und veränderter historischer Zusammenhänge - und dazu gehören dann auch die Naturwissenschaften. Wenn Monroe Beardsley und später auch Paul Ricœur für die Interpretation von Gedichten festlegten, alle möglichen Konnotationen müssten berücksichtigt werden und ein Gedicht bedeute alles, was es bedeuten

28 Vgl. zum Folgenden auch Vanderbeke: Theoretische Welten, S. 78-81.

29 Vgl. Stephen Jay Gould: Ladders and Cones. Constraining Evolution by Canonical Icons. In: Hidden Histories of Science. Hrsg. von R. B. Silvers. New York: New York Review, 1995. S. 37-67, hier S. 42 und passim.

30 Max Black: Metaphor. In: Proceedings of the Aristotelian Society. New Series 55 (1954-1955).

S. 273-294, hier S. 288. 
könne, ${ }^{31}$ dann geht es dabei um die Reichhaltigkeit der Bedeutungspotentiale und schließlich um die Unabschließbarkeit des Interpretationsprozesses.

An dieser Stelle möchte ich noch einmal zu der Passage aus Dante und zu der Vision der drei farbigen Kreise zurückkehren. Wie erwähnt, ist die Bedeutung dieses Bildes zunächst einmal eher einfach und hinter den drei Kreisen ist die Trinität zu erkennen. Daraus kann man aber natürlich auch noch mehr machen, und die Interpretationen der Passage können recht umfangreich werden. Bei der Exegese des Textes findet man dann gelegentlich auch Lesarten, bei denen aus den drei Farben eine weiße Neutralität folgt. So schrieb William Bishop 1910: „Thus the white light of colorless ,tri-unity ' [...] takes on the varied hues of those concrete personal distinctions which exist in what we may perhaps reverently speak of as the Divine Family“. ${ }^{32}$ Analog dazu heißt es dann neuerlich auf einer eher mystischen Website: „Wie das sichtbare weisse Licht besteht laut Dante also auch das Urlicht aus verschiedenen Farben, die verschiedene psychische Eigenschaften darstellen. “33

Das ist interessant, wenn man in Betracht zieht, dass zu Dantes Zeit die Farbenlehre vermutlich noch nicht so weit gediehen war, dass Weiß als das Ergebnis einer Mischung der Grundfarben angesehen wurde. ${ }^{34}$

Aber man kann natürlich auch noch weiter gehen. So veröffentlichte Bob Estes, laut seiner Website Physiker, zu der Passage aus dem Paradiso einen OnlineBlog mit dem Titel „Dante's Heavenly Vision and the Physics of the Proton“. Darin führt er eben die Aspekte an, die Klaus Mecke in seinem Beitrag zu den Farben der Quarks behandelt, und er kommt am Ende zu der Frage, ob es denn zufällig sein kann, dass eine solche Analogie zwischen der Physik und der Dreifaltigkeit besteht:

Do the similarities between Dante's poetic vision of the Christian doctrine of the Triune God and our modern, well-established theory of the tri-quark proton amount to more than a curious historical coincidence? Does this analogy go beyond the merely amusing to the deeply significant?

31 Vgl. Monroe C. Beardsley: Aesthetics. Problems in the Philosophy of Criticism. New York: Harcourt, Brace, 1958. S. 144; und Paul Ricœur: Metaphor and the Main Problem of Hermeneutics. In: New Literary History 6.1 (Themenheft: On Metaphor. Herbst 1974). S. 95-110, hier S. 104.

32 William Samuel Bishop: The Development of Trinitarian Doctrine in the Nicene and Athanasian Creeds. A Study in Theological Definition. New York u. a.: Longmans Green, 1910. S. 17.

33 Leonhard Heinzmann: Nirvana, ein anderes Wort für Heiliger Geist. www.ieao.de/nirvana.htm. Private Homepage, undat. Eintrag (14. April 2015).

34 Ich möchte mich an dieser Stelle bei Rudolf Suntrup, dem Mitherausgeber des Lexikons der Farbenbedeutungen im Mittelalter, bedanken, der mir darüber freundlicherweise Auskunft gegeben hat. 


\author{
Of course not. \\ It can't, can it? \\ Maybe. \\ How could it not? \\ But that's crazy. \\ Isn't it? ${ }^{35}$
}

Für Estes, einen gläubigen Physiker, ist das natürlich kein Zufall, und die Photonen könnten demnach eine göttliche Botschaft und Aufforderung an die zweifelnden Wissenschaftler sein, in der Natur das Bild Gottes zu erkennen. Dantes erleuchtete Dichtung wird sozusagen verifiziert; seine Metapher erweist sich als inspirierte Vision und gleichzeitig als Antizipation wissenschaftlicher Erkenntnis.

Es ist durchaus nicht meine Absicht, solche Lesarten lächerlich zu machen selbstverständlich ist es möglich, literarische Texte im Licht späterer Erkenntnisse neu zu interpretieren und in ihnen gelegentlich auch Aspekte zu finden, die dem Autoren nicht bekannt gewesen sein können. Manche Autoren eignen sich dafür besser als andere, und es gibt inzwischen Interpretationen von Borges im Kontext der Chaostheorie, der Feldtheorie, der Quantenphysik und der Relativitätstheorie. Bei Beckett wurden ebenfalls Chaostheorie und Quantenphysik, aber auch ,Schwarze Löcher' gefunden, und bei Joyce gilt sowieso, dass wer sucht, auch findet. ${ }^{36}$

Einer der Gründe, die solche Interpretationen begünstigen, liegt wohl darin, dass in der Literatur nicht ähnlich stringente Kriterien für die Schönheit der untersuchten Phänomene und ihre Interpretation angenommen werden wie in der Physik. An dieser Stelle möchte ich noch einmal auf Steven Weinberg zurückkommen. Er schreibt zur Schönheit in physikalischen Theorien:

The kind of beauty that we find in physical theories is of a very limited sort. It is, as far as I have been able to capture it in words, the beauty of simplicity and inevitability - the beauty of perfect structure, the beauty of everything fitting together, of nothing being changeable, of logical rigidity. ${ }^{37}$

Eine ähnliche Bewertung findet sich auch bei Graham Farmelo in der Einleitung $\mathrm{zu}$ seinem Buch It Must Be Beautiful. Great Equations of Modern Science. Er schreibt dort:

35 Bob Estes: Dante's Heavenly Vision and the Physics of the Proton. www.onscreen-scientist. $\mathrm{com} / \mathrm{p}=49$. Private Homepage On-Screen Scientist, Eintrag vom 13. März 2009 (14. April 2015).

36 Vgl. Vanderbeke: Theoretische Welten, S. 227.

37 Weinberg: Dreams, S. 119. 
Much like a work of art, a beautiful equation has among its attributes much more than mere attractiveness - it will have universality, simplicity, inevitability and an elemental power. [...] An additional quality of a good scientific equation is that it has utilitarian beauty. It must tally with the result of every relevant experiment and, even better, make predictions that no one has ever made before. ${ }^{38}$

G. H. Hardy erklärte schon 1940 für mathematische Theorien: „Beauty is the first test: there is no permanent place in the world for ugly mathematics“ “, ${ }^{39}$ und nennt dann als Kriterien: „a very high degree of unexpectedness, combined with inevitability and economy“, 40 wobei die ,economy“ hier anscheinend den Platz der „simplicity“ einnimmt. Quasi die gleichen Kriterien wie bei den hier angeführten Wissenschaftlern werden auch von Thomas S. Kuhn genannt - allerdings geht es bei ihm nicht um die Schönheit von Theorien, sondern um Theoriewahl im Allgemeinen. Er nennt fünf Eigenschaften für die Beurteilung von Theorien: Tatsachenkonformität, Widerspruchsfreiheit, Reichweite, Einfachheit und Fruchtbarkeit. ${ }^{41}$

Es scheint, als hätte Ockhams Rasiermesser nichts an seiner Bedeutung verloren und dabei inzwischen auch eine ästhetische Komponente erhalten. Der Begriff der Einfachheit ist hier allerdings ganz sicher nicht im umgangssprachlichen Sinne zu verstehen oder gar mit Trivialität oder Banalität gleichzusetzen. Er bekommt seine Bedeutung, so wie ich es verstehe, in der Verbindung mit den anderen Eigenschaften, d.h., es geht um eine Einfachheit, die gleichzeitig reichhaltig und anschlussfähig ist, also vielseitige Verbindungen zulässt. Philippe Blanchard beschreibt dies einerseits als „riesige Anzahl an Anwendungen“ und andererseits als „Kompaktheit“. ${ }^{42}$ In der zunächst einfachen Theorie oder Gleichung liegt also das Potential für Komplexität, $d$. h. aus wenigen klaren Prinzipien lässt sich eine Vielzahl an Phänomenen ableiten und - so wird es für eine selbstverständlich schöne Theory of Everything erhofft - schließlich das gesamte Universum erklären. Während also die naturwissenschaftliche Metapher, wie oben ausgeführt, eine Tendenz hat, sich mit der Zeit zu verengen, zeichnet sich die schöne physikalische Gleichung eben auch dadurch aus, dass sie weitreichende Möglichkeiten

38 Graham Farmelo: Foreword: It must be beautiful. In: ders.: It Must Be Beautiful. Great Equations of Modern Science. London: Granta, 2002. S. XIV.

39 Harold Hardy: A Mathematician's Apology. Cambridge: Cambridge UP, 1940. S. 14.

40 Ebd., S. 29. Herv. im Orig.

41 Vgl. Thomas S. Kuhn: Objektivität, Werturteil und Theoriewahl. In: ders.: Die Entstehung des Neuen. Frankfurt a. M.: Suhrkamp, 1978. S. 423.

42 Philippe Blanchard: Chaotische Bemerkungen eines theoretischen Physikers zur Ästhetik. In: Ästhetik der Wissenschaft. Interdisziplinärer Diskurs über das Gestalten und Darstellen von Wissen [Zeitschrift für Ästhetik und Allgemeine Kunstwissenschaft. Sonderheft 7]. Hrsg. von Wolfgang Krohn. Hamburg: Meiner, 2006. S. 167-173, hier S. 169. 
nicht nur eröffnet, sondern gleichsam realisiert. Indem die Gleichung zwei bisher unverbundene Phänomene als einander synonym erkennt, fordert sie ein neues Verständnis sämtlicher physikalischer Gegebenheiten, die in Relation zu diesen Phänomenen stehen - und so ist es durchaus zu verstehen, wenn es Heisenberg bei dem Gedanken schwindlig wurde, dass er nun all jenen Strukturen nachgehen sollte, die seine neue Theorie berührte.

An dieser Stelle möchte ich zur Literatur übergehen, wobei ich zunächst den Blick von außerhalb auf die Disziplin heranziehen möchte. Hier noch einmal Steven Weinberg, der zunächst auf die Unzugänglichkeit - er nennt es Esoterik physikalischer Theorien eingeht und dann schreibt:

Physicists generally do not like the fact that our theories are so esoteric. On the other hand, I have occasionally heard artists talk proudly about their work being accessible only to a band of cognoscenti and justify this attitude by quoting the example of physical theories like general relativity that also can be understood only by initiates. Artists like physicists may not always be able to make themselves understood by the general public, but esotericism for its own sake is just silly. ${ }^{43}$

Der letzte Satz trifft natürlich in das Herz der Literaturtheorie des zwanzigsten Jahrhunderts, denn hier wird der bewussten Schwierigkeit literarischer Werke überwiegend wohlwollende Aufmerksamkeit geschenkt. Als Ausgangspunkt für diese Entwicklung könnte Viktor Shklovskys programmatischer Aufsatz „Kunst als Verfahren“ von 1917 angesehen werden, in dem er als das Grundprinzip der Kunst und besonders der Dichtung die Verfremdung beschrieb. Es geht dabei tatsächlich um eine Art der Erschwerung und Komplizierung um ihrer selbst willen. Da die Kunst auf ihre Wahrnehmung, also die ästhetische Erfahrung, abzielt, sind Elemente und Textstrategien, die diesen Prozess verlängern oder ausdehnen, grundsätzlich im Interesse der Kunst sowie auch des Rezipienten. ${ }^{44}$ Das Werk, das sich dem einfachen Zugang entzieht und von der Leserschaft besondere Anstrengungen verlangt, ist hier einem eingängigen und leicht verständlichen Text grundsätzlich vorzuziehen. In einem literarischen Manifest aus dem Umkreis von James Joyce heißt es dann auch ganz deutlich: „The writer expresses. He does not communicate“, und: „The plain reader be damned““.45 Ähnliche Überlegungen und Prinzipien durchziehen die Literaturtheorie des zwanzigsten Jahrhunderts. Und wenn auch zu Beginn der Postmoderne kritische

43 Weinberg: Dreams, S. 119.

44 Vgl. Viktor Shklovsky: Art as Technique [1917]. Übers. von Lee T. Lemon und Marion J. Reis. In: Modern Criticism and Theory. Hrsg. von David Lodge. London: Longman, 1988. S. 16-30, hier S. 20.

45 Richard Ellman: James Joyce [1959]. Überarb. Ausgabe. Oxford u. a.: Oxford UP, 1983. S. 588. 
Stimmen zum Angriff auf die Kirche und Priesterschaft einer überkomplexen modernen Hochliteratur aufgerufen haben - z. B. Leslie Fiedler 1969 in seinem Aufsatz „Cross the Border - Close the Gap“ ${ }^{46}$, so waren die darauf folgenden Texte von John Barth, Thomas Pynchon, Robert Coover oder David Foster Wallace auch nicht unbedingt zugänglicher als die von James Joyce, T. S. Eliot oder Virginia Woolf.

Die programmatische Annahme einer Verwiderspenstigung des Textes findet sich in einer Vielzahl von theoretischen Ansätzen. Gadamer z. B. schreibt von dem Anstoß, den wir an einem Text nehmen - an der Andersartigkeit der Sprache oder der inhaltlichen Vormeinungen. Erst dieser Anstoß begründet Gadamer zufolge den komplexen und letztlich unabschließbaren Prozess, den wir Verstehen nennen. ${ }^{47}$ Dass ein solches Verstehen rein hypothetisch einmal stattfinden könnte, ist aber keinesfalls bei allen Theoretikern gewährleistet. Über einigen Literaturtheorien steht quasi in großen Buchstaben: „Am Anfang war das Wort und das Wort wird grundsätzlich missverstanden.“ Die apodiktische Feststellung „Every interpretation is a misinterpretation, every reading is a misreading" ist inzwischen schon so verbreitet, dass sie im Internet wahlweise und nicht ganz ohne Berechtigung Jonathan Culler, Harold Bloom, Roland Barthes, Paul de Man, Michel Foucault, Jacques Derrida, Umberto Eco oder Derek Attridge zugeschrieben wird und diese Liste ist vermutlich nicht vollständig. Der literarische Text entzieht sich also nicht nur dem einfachen, sondern letztlich jedem Verstehen, wobei dies jedoch auch als eine Befreiung verstanden werden kann.

Eine Ästhetik, die auf einer Erschwerung des Verstehens beruht oder diese als wesentlichen Bestandteil integriert, kommt sicher dem literaturwissenschaftlichen Interesse entgegen, da sich daraus theoretische Überlegungen und Konzeptionen erarbeiten lassen, durch die sich die für uns zumeist maßgeblichen Texte erfassen und beschreiben lassen. Eine solche Ästhetik steht aber nicht nur im Widerspruch zu kognitionswissenschaftlichen Untersuchungen, sondern auch zu der tatsächlichen Rezeption von Literatur. Unzweifelhaft gibt es eine Vielzahl von Menschen, die eine beträchtliche Komplexität in literarischen Texten zu schätzen wissen. Es wäre aber problematisch, diese als maßgeblich für das menschliche ästhetische Empfinden per se zu setzen, wie es z. B. auch falsch wäre, den natürlichen menschlichen Bewegungstrieb am Leistungssportler zu erforschen. Und es ist hier vielleicht auch nicht unerheblich, dass quasi alle bedeutenden künstlerischen Erneuerungen zunächst einmal auf massive Ablehnung stießen und selbst

46 Vgl. Leslie Fiedler. Cross the Border - Close the Gap [1969]. In: ders.: Cross the Border - Close the Gap. New York: Stein and Day, 1972. S. 64.

47 Vgl. Hans-Georg Gadamer: Wahrheit und Methode [1960]. Tübingen: J. C. B. Mohr, 1975. S. 252. 
vom ,gebildeten' Publikum erst einmal neu ,gelernt ' werden mussten, bevor sie auf ästhetisches Wohlgefallen trafen - das Konzept einer ,mere exposure theory“ beschreibt dieses Phänomen einer ästhetischen Eingewöhnung. ${ }^{48}$

Wenn wir uns der Frage zuwenden, warum wir an Geschichten Vergnügen empfinden, und warum dies auch schon bei Kindern und sogar Kleinkindern der Fall ist, also bei den allerersten Anfängen des literarischen Interesses, dann können wir feststellen, dass Aspekte wie der Wiedererkennungseffekt weitaus wichtiger sind als eine Widerspenstigkeit der Lektüre, dass Eingängigkeit eher ankommt als Verfremdung. Und dabei müssen wir uns gar nicht auf Kinder beschränken. Michael Bérubé hat in einem sehr witzigen Artikel mit dem Titel „Teaching Postmodern Fiction Without Being Sure that the Genre Exists“ darauf hingewiesen, dass der weitaus größte Teil der Literatur im Zeitalter der Postmoderne weder postmodern noch modern ist, sondern eher prämodernen Erzählformen folgt ${ }^{49}$ Diese Literatur - von Groschenheften über Harlekin Romances bis zu Historienschinken oder den neueren Verschwörungsromanen von Dan Brown - mag banal sein oder trivial oder Massenware oder gelegentlich auch mal richtig schöner Schund, aber sie findet hingebungsvolle Anhängerschaften, die sich bei jeder Neuerscheinung an den neuen Varianten des Wohlbekannten erfreuen.

In einem sehr interessanten Artikel aus dem Forschungsbereich der neuronalen Psychologie behandeln die Autoren Rolf Reber, Norbert Schwarz und Piotr Winkielman das Thema „Processing Fluency and Aesthetic Pleasure“. Das Gefühl für Schönheit wird dabei definiert als ,a pleasurable subjective experience that is directed toward an object and not mediated by intervening reasoning “. ${ }^{50} \mathrm{Die}$ empirisch begründete These des Artikels lautet dann: „The more fluently the perceiver can process an object, the more positive is his or her aesthetic response." ${ }^{11}$ Wohlgefallen wird also von einem Reiz, einer Wahrnehmung hervorgerufen, die eingängig ist und sich leicht und ohne inhärente Widerstände quasi fließend verarbeiten lässt. Der Artikel beschäftigt sich zwar vornehmlich mit visueller Ästhetik, es werden aber auch Überlegungen zum Vergnügen an Musik angeführt und die Autoren schreiben zudem, ,our arguments apply as well to conceptual fluency, or the ease of mental operations concerned with stimulus meaning and its relation

48 Vgl. James Cutting: Gustave Caillebotte, French Impressionism, and mere exposure. In: Psychonomic Bulletin and Review 10.2 (2003). S. 319-343 und passim.

49 Michael Bérubé: Teaching Postmodern Fiction Without Being Sure that the Genre Exists. In: The Chronicle of Higher Education. 19. Mai 2000. B4-B5.

50 Rolf Reber u. a.: Processing Fluency and Aesthetic Pleasure. Is Beauty in the Perceiver's Processing Experience? In: Personality and Social Psychology Review 8.4 (2004). S. 364-382, hier S. 365 .

51 Ebd. 
to semantic knowledge structures." 52 Damit wäre dann wohl auch die Literatur miteinbegriffen. Diese Ergebnisse widersprechen natürlich den oben zitierten Forderungen nach einer Widerspenstigkeit der Information gegen ihre Verarbeitung, wie sie in der modernen und inzwischen postmodernen Literaturtheorie quasi zur conditio sine qua non des literarischen Kunstwerks erhoben worden sind. Damit soll keinesfalls den literaturtheoretischen Konzepten eines ganzen Jahrhunderts widersprochen werden, es soll nur festgestellt werden, dass sie sich auf einen spezifischen Literaturbegriff bezogen und die weniger interessante Massenliteratur nur sehr nebenbei zur Kenntnis nahmen.

Es lässt sich aber nun auch die Frage stellen, ob es nicht auch in der Literatur Strukturen und Phänomene gibt, die mit den Kriterien, die oben für die Physik beschrieben wurden, in Einklang zu bringen sind. Ich denke, dass dies der Fall ist.

An dieser Stelle lohnt es sich, noch einmal auf die Metapher zurückzukommen. Gerade durch ihre Reichweite und potentiell unabschließbare Interpretierbarkeit findet sich hier eine Analogie zu der schönen Theorie bzw. zur schönen Gleichung. Bekanntlich sind Metaphern zumeist eine bildliche Reduktion des Abstrakten auf das Konkrete, und sie erlauben daher einen Zugang zum Komplexen durch ein eher eingängiges Bild. Gleichzeitig aber besitzt die gelungene Metapher durch ihre vielfältige Interpretierbarkeit selbst eine Tendenz zur Komplexität, sie hat die Fähigkeit, sich zu entfalten. Wenn, wie oben ausgeführt, die schöne Gleichung überraschende Perspektiven eröffnet, die in ihrer Klarheit und Stringenz erfordern, die Welt und die in ihr ablaufenden Prozesse völlig neu zu sehen, so vermag die gelungene literarische Metapher - die ja auch ein Gleichheitsverhältnis postuliert - immer neue Lesarten hervorzubringen und ermöglicht damit auch neue und unerwartete produktive Sichtweisen auf unsere Erfahrungswelt. Dabei sind die Möglichkeiten, die sich aus dem eigentlich einfachen Bild ergeben, unabschließbar. Hans Blumenberg hat in einigen seiner Bücher verfolgt, wie einzelne Metaphern, Tropen oder kurze Parabeln im Laufe der Geschichte immer neue Bedeutungen annahmen und in unterschiedlichster, teilweise auch gegensätzlicher Weise ausgelegt wurden. Borges schreibt ganz in diesem Sinne am Anfang seines Essays „Die Sphäre Pascals“: „Vielleicht ist die Universalgeschichte die Geschichte einiger weniger Metaphern“, und schließt den Essay mit den Worten: „Vielleicht ist die Universalgeschichte die Geschichte der unterschiedlichen Betonung einiger weniger Metaphern." ${ }^{53}$

52 Ebd., S. 366. Herv. im Orig.

53 Jorge Luis Borges: Inquisitionen [Otras inquisitiones]. Übers. von Karl August Horn und Gisbert Haefs. Frankfurt a. M.: Fischer, 1992. S. 15 und 18. 
Im Kontext der Literatur und des Erzählens könnten darüber hinaus auch bestimmte Motive und Mythologeme als Analogie einer schönen, ,einfachen' Theorie mit weitreichenden Implikationen angesehen werden. Der Mythos ist in mancher Hinsicht der Metapher nicht unähnlich, indem eine konkrete Erzählung abstrakte Inhalte vermittelt. Wie die Metapher ist der Mythos unendlich anschlussfähig und interpretierbar, er ist eingängig und doch ist in ihn eine beträchtliche Komplexität eingefaltet. Eine genaue Betrachtung würde hier zu weit führen, daher möchte ich zu meinem letzten Punkt kommen, der noch einmal an die Interpretierbarkeit der Tropen anschließt.

Alle bisherigen Ausführungen zur Ästhetik in der Physik bezogen sich auf schöne Theorien, die als produktiv, nützlich und zutreffend erkannt und daher auch von der relevanten Forschergemeinschaft angenommen wurden. Der Satz, dass Schönheit und Wahrheit zusammenfallen, wäre damit also mehr oder weniger bestätigt. Wie steht es jedoch mit Theorien, die schließlich verworfen wurden? Die Wissenschaftsgeschichte ist ja die Geschichte von Theorien, die einander ablösen, und nicht die Geschichte von solchen, die für alle Zeiten bestehen bleiben. Lässt sich auch bei ihnen möglicherweise eine ästhetische Komponente feststellen - sozusagen eine Schönheit der gescheiterten Theorien?

Ich möchte vorschlagen, dass dies wirklich der Fall ist. Es gibt meines Erachtens Theorieansätze und Erklärungsmuster, die die Wissenschaftsgeschichte durchziehen und in den unterschiedlichsten Varianten immer wieder auftauchen, nur um letztlich auch immer wieder verworfen zu werden. In diesem Sinne schreibt Borges in seinem Essay „Quevedo“: „Es gibt in der Geschichte der Philosophie Lehren, die vermutlich falsch sind, die aber mit dunkler Zauberkraft auf die Phantasie der Menschen wirken". ${ }^{54}$ Sie tauchen vornehmlich in Zeiten auf, in denen Paradigmenwechsel stattfinden, die jeweilige Wissenschaft sich also mit ernsthaften Widersprüchen zu dem bestehenden Paradigma konfrontiert sieht und eine Neukonzeption vorgenommen werden muss. Thomas S. Kuhn hat für diese Zeiten eine Vervielfältigung oder sogar ein Wuchern theoretischer Spekulationen beschrieben, durch die ein Weg aus der momentanen Krise gefunden werden soll. ${ }^{55}$

Ganz oben auf meiner Liste derartiger Erklärungsmuster steht die Annahme einer Selbstähnlichkeit, dass also innerhalb eines größeren Zusammenhangs ein Ähnlichkeitsverhältnis zwischen dem Teil und dem Ganzen besteht. ${ }^{56}$ Alternative

54 Borges: Inquisitionen, S. 47-48.

55 Vgl. Thomas S. Kuhn: The Structure of Scientific Revolutions [1962]. 2. überarb. Ausg. Chicago: University of Chicago Press, 1970. S. 70-71 und 87.

56 Die diesbezüglichen Überlegungen habe ich schon anderweitig ausführlicher behandelt. Vgl. z. B. Vanderbeke: Of Parts and Wholes: Self-similarity and Synecdoche in Science, Culture and 
und leicht abweichende Begriffe wären hier der Organizismus bzw. der Holismus, bei denen ebenfalls ein Analogieverhältnis zwischen dem Teil und dem Ganzen besteht.

Derartige Erklärungsmuster sind sehr alt und letztlich auch schon im Mythos zu finden, wenn das Universum aus dem Körper eines Urwesens geformt wird und damit also eine Ähnlichkeit zwischen dem Menschen und dem Kosmos besteht. Dies findet sich dann wieder in der Analogie von Mikrokosmos und Makrokosmos, wobei hier gleich eine Vielzahl von Ebenen angenommen wurde - von den Sternen und Planeten, die noch keine Himmelskörper nach unserer Vorstellung waren, zu der Erde, zum Staat und schließlich zum Menschen und seinem Körper, wobei gelegentlich auch noch kleinere Systeme vorgeschlagen wurden. Solche Gedanken können leicht wiederbelebt werden: Als Niels Bohr 1922 den Nobelpreis erhielt, sagte er über die Struktur des Atoms, man könnte darin eine deutliche Ähnlichkeit zu dem System der Planeten in unserem eigenen Sonnensystem erkennen. ${ }^{57}$ Max Born schreibt dazu, ganz in dem hier vertretenen Sinne (und auch im Sinne des oben angeführten Zitats von Borges):

Eine der merkwürdigsten und anziehendsten Ergebnisse der Bohrschen Atomtheorie ist die Vorstellung, daß die Atome Planetensysteme im Kleinen sind. Der Gedanke, daß sich die Gesetze des Makrokosmos in der kleinen irdischen Welt widerspiegeln, übt offensichtlich einen großen Zauber auf das menschliche Gemüt aus; bildet er doch eine Wurzel jenes Aberglaubens (der so alt ist wie die Geschichte des Geistes), daß die Schicksale der Menschen aus den Sternen gelesen werden können. ${ }^{58}$

Born kommt zu dem Schluss, „daß die Ähnlichkeit der Atome mit den Planeten ihre Grenzen hat“; 59 das eingängige Bild, das auf uns einen besonderen Reiz hat, musste schon ein Jahr nach Bohrs Rede wieder verworfen werden. Analoge Ansätze, die eine Ähnlichkeit zwischen dem Teil und dem Ganzen annehmen, finden sich gerade zu Beginn des zwanzigsten Jahrhunderts an vielen Stellen, nicht nur in der Physik, sondern auch in der Biologie, Geschichte oder Philosophie. Alfred North Whitehead schreibt 1929: „[J]edes wirkliche Einzelwesen an sich [lässt] sich nur als ein organischer Prozess beschreiben. Es wiederholt im Mikrokosmos, was

Literature. In: Reconstruction 4.4 (Herbst 2004). http://reconstruction.eserver.org/Issues/044/ vanderbeke.htm (14. April 2015). Es folgt hier daher nur ein kurzer Überblick.

57 Vgl. Niels Bohr: Nobel Prize in Physics Award Address [1922]. In: The World of Physics. Bd. 2. Hrsg. von J. H. Weaver. New York: Simon and Schuster, 1987. S. 315-338, hier S. 316.

58 Max Born: Quantentheorie und Störungsrechnung. In: Naturwissenschaften 27 (1923). S. 537542, hier S. 537.

59 Ebd., S. 542. 
das Universum im Makrokosmos ist“". ${ }^{0}$ Von dort - oder genauer gesagt: von der Philosophie Henri Bergsons, auf die Whitehead verweist - zieht sich eine Linie, die zu David Bohms Konzept einer dem Universum zugrunde liegenden Ordnung führt, in der wie bei einem Hologramm jeder Teil untrennbar mit dem Ganzen verwoben ist und auch Information über das Ganze enthält. ${ }^{61}$ Von Bohm geht die Linie dann weiter zu Ilya Prigogines Überlegungen zu einem organischen Werden und einer spontanen Selbstorganisation in speziellen chemischen und physikalischen Prozessen. ${ }^{62}$

In der Rhetorik lässt sich das verwandte Phänomen der Synekdoche ausmachen, bei der ein Teil auf das Ganze verweist; in der Literatur finden sich analog dazu Verschachtelungen, die u. a. als Russian dolls, Chinese boxes oder mise en abyme bezeichnet werden. In jedem der Fälle findet sich eine Art Selbstähnlichkeit, Werner Wolf definiert dies als „,die Spiegelung einer Makrostruktur eines literarischen Textes in einer Mikrostruktur innerhalb desselben Textes"63, wobei sich diese Spiegelung auf mehreren Ebenen fortsetzen kann. Ich halte es für durchaus denkbar, dass hier ein ästhetisches Prinzip vorliegt, das im Denken des Menschen verankert ist, eventuell sogar evolutionär erworben wurde, und das daher in den unterschiedlichen Zugängen zur Wirklichkeit immer wieder auftaucht. In der Literatur, die sich nicht an der Realität beweisen muss, ist es ein beständiges Phänomen, eine Textstrategie, die sich in narrativen Mustern, einzelnen Motiven oder spezifischen Strukturen niederschlägt. In der Naturwissenschaft werden derartige Erklärungsmuster - soweit ich es überblicken kann - zumeist wieder verworfen (eine Ausnahme bildet die Chaostheorie oder die nicht-lineare Dynamik, in der selbstähnliche Fraktale eine wesentliche Rolle spielen). Aber vielleicht stoßen die Wissenschaftler ja in der Zukunft auch auf eine Theory of Everything, die auf selbstähnlichen Prinzipien und Strukturen basiert, und bestätigen damit noch einmal, dass Schönheit und Wahrheit zusammenfallen.

60 Alfred North Whitehead: Prozess und Realität. Entwurf einer Kosmologie [Process and Reality. An Essay in Cosmology, 1929]. Übers. von Hans Günther Holl. Frankfurt a. M.: Suhrkamp, 1987. S. 397.

61 Vgl. David Bohm: Wholeness and Implicate Order [1980]. London und New York: Routledge, 1995. S. 145-147.

62 Vgl. Ilya Prigogine und Isabelle Stengers: Dialog mit der Natur. Neue Wege naturwissenschaftlichen Denkens [Order out of Chaos. Man's New Dialogue with Nature, 1980]. Übers. von F. Griese. München: Piper, 1981. S. 98-104.

63 Werner Wolf: Ästhetische Illusion und Illusionsdurchbrechung in der Erzählkunst. Tübingen: Niemeyer, 1993. S. 296. 


\section{Literatur}

Beardsley, Monroe C: Aesthetics: Problems in the Philosophy of Criticism. New York: Harcourt, Brace, 1958.

Bérubé, Michael: Teaching Postmodern Fiction Without Being Sure that the Genre Exists. In: The Chronicle of Higher Education. 19. Mai 2000. B4-B5.

Bishop, William Samuel: The Development of Trinitarian Doctrine in the Nicene and Athanasian Creeds. A Study in Theological Definition. New York u. a.: Longmans, Green and Co, 1910.

Black, Max. Metaphor. In: Proceedings of the Aristotelian Society. New Series 55 (1954-1955). S. 273-294. Auch unter: www.stanford.edu/ eckert/PDF/Black1954.pdf (14. April 2015).

Blanchard, Philippe: Chaotische Bemerkungen eines theoretischen Physikers zur Ästhetik. In: Ästhetik der Wissenschaft. Interdisziplinärer Diskurs über das Gestalten und Darstellen von Wissen [Zeitschrift für Ästhetik und Allgemeine Kunstwissenschaft. Sonderheft 7]. Hrsg. von Wolfgang Krohn. Hamburg: Meiner, 2006. S. 167-173.

Bloom, Harold: The Anxiety of Influence [1973]. Oxford u. a.: Oxford University Press, 1997. Bohm, David: Wholeness and Implicate Order [1980]. London und New York: Routledge, 1995. Bohr, Nils: Nobel Prize in Physics Award Address [1922]. In: The World of Physics. Bd. 2. Hrsg. von J. H. Weaver. New York: Simon and Schuster, 1987. S. 315-338.

Borges, Jorge Luis: Inquisitionen [Otras inquisitiones]. Übers. von Karl August Horn und Gisbert Haefs. Frankfurt a. M.: Fischer, 1992.

Born, Max: Quantentheorie und Störungsrechnung. In: Naturwissenschaften 27 (1923). S. 537-542.

Braungart, Wolfgang, und Silke Jakobs: Staunen und Hingabe. Zur Ästhetik des Wissens seit dem 18. Jahrhundert. In: Ästhetik der Wissenschaft. Interdisziplinärer Diskurs über das Gestalten und Darstellen von Wissen [Zeitschrift für Ästhetik und Allgemeine Kunstwissenschaft. Sonderheft 7]. Hrsg. von Wolfgang Krohn. Hamburg: Meiner, 2006. S. 201-218.

Breidbach, Olaf: Neuronale Ästhetik. München: Wilhelm Fink, 2013.

Burke, Edmund: A Philosophical Enquiry into the Origin of our Ideas of the Sublime and Beautiful [1757]. Hrsg. von Adam Phillips. Oxford u. a..: Oxford University Press, 1990.

Cutting, James: Gustave Caillebotte, French Impressionism, and mere exposure. In: Psychonomic Bulletin and Review 10.2 (2003). S. 319-343.

Dante Alighieri: La vita nuova/La divina commedia. Ital./dt., übers. v. Richard Zoozmann. Augsburg: Weltbild, 1994.

Devlin, Keith: The Maths Gene: Why everyone has it but most people don't use it. London: Phoenix, 2001.

Dutton, Dennis: The Art Instinct. Beauty, Pleasure, and Human Evolution. New York u. a.: Bloomsbury, 2010.

Dyson, Freeman: Is Science Mostly Driven by Ideas or by Tools? In: Science 338 (14. Dezember 2012). S.1426-1427. Dt. Übers.: Theorie und Werkzeug. In: Süddeutsche Zeitung. 14. Dezember 2012. S. 18.

Ellman, Richard: James Joyce [1959]. Überarb. Ausg. Oxford u. a.: Oxford University Press, 1983.

Estes, Bob: Dante's Heavenly Vision and the Physics of the Proton. www.onscreen-scientist. $\mathrm{com} / \mathrm{p}=4$ 49. Private Homepage On-Screen Scientist, Eintrag vom 13. März 2009 (14. April 2015).

Farmelo, Graham: Foreword: It must be beautiful. In: It Must Be Beautiful. Great Equations of Modern Science. London und New York: Granta, 2002. S. xi-xviii. 
Fiedler, Leslie: Cross the Border - Close the Gap [1969]. In: ders.: Cross the Border-Close the Gap. New York: Stein and Day, 1972. S. 61-85.

Gadamer, Hans-Georg: Wahrheit und Methode [1960]. Tübingen: J. C. B. Mohr, 1975.

Gell-Mann, Murray: The Quark and the Jaguar. New York: W. H. Freeman, 1994.

Gould, Stephen Jay: Ladders and Cones. Constraining Evolution by Canonical Icons. In: Hidden Histories of Science. Hrsg. von R. B. Silvers. New York: New York Review, 1995. S. 37-67.

Hardy, Godfrey Harold: A Mathematician's Apology. Cambridge: Cambridge University Press, 1940. Auch unter: http://www.math.ualberta.ca/mss/misc/A\%20Mathematician\%27s \%20Apology.pdf (14. April 2015).

Hayles, N. Katherine: Introduction: Complex Dynamics in Literature and Science. In: Chaos and Order. Complex Dynamics in Literature and Science. Hrsg. von N. Katherine Hayles. Chicago und London: University of Chicago Press, 1991. S. 1-33.

Heinzmann, Leonhard: Nirvana, eine anderes Wort für Heiliger Geist. www.ieao.de/nirvana. htm. Private Homepage, undat. Eintrag (14. April 2015).

Heisenberg, Werner: Der Teil und das Ganze [1969]. München: dtv, 1987.

Heisenberg, Werner: Die Bedeutung des Schönen in den exakten Naturwissenschaften. In: ders.: Schritte über Grenzen. Erw. Neuausg. München: Piper, 1977.

Joyce, James: Finnegans Wake [1939]. London: Penguin, 1975.

Keats, John: Ode on a Grecian Urn. In: Romantic Poetry. An Annotated Anthology. Hrsg. von Michael O’Neill und Charles Mahoney. Malden, MA: Blackwell, 2008. S. 450-451.

Kuhn, Thomas S.: The Structure of Scientific Revolutions [1962]. 2. durchgesehene Ausg. Chicago: University of Chicago Press, 1970.

Kuhn, Thomas S.: Objektivität, Werturteil und Theoriewahl. In: ders.: Die Entstehung des Neuen. Frankfurt a. M.: Suhrkamp, 1978. S. 421-445.

Mazur, Barry: Imagining Numbers (Particularly the Square Root of Minus Fifteen). London: Penguin, 2004.

Medewar, Peter: Is the Scientific Paper Fraudulent? In: The Saturday Review. 1. August 1964. S. 42-43.

Moore, Walter J.: A Life of Erwin Schrödinger. Cambridge: Cambridge University Press, 1994.

Mozzochi, Charles J.: The Fermat Diary. Providence, RI: American Mathematical Society, 2000.

Penrose, Roger (mit Abner Shimony, Nancy Cartwright und Stephen Hawking): The Large, the Small, and the Human Mind. Cambridge: Cambridge University Press, 1999.

Prigogine, Ilya, und Isabelle Stengers: Dialog mit der Natur. Neue Wege naturwissenschaftlichen Denkens [Order out of Chaos. Man's New Dialogue with Nature, 1980]. Übers. von F. Griese. München und Zürich: Piper, 1981.

Reber, Rolf, Norbert Schwarz und Piotr Winkielman: Processing Fluency and Aesthetic Pleasure. Is Beauty in the Perceiver's Processing Experience? In: Personality and Social Psychology Review 8.4 (2004). S. 364-382.

Ricœur, Paul: Metaphor and the Main Problem of Hermeneutics. In: New Literary History 6.1 (Themenheft: On Metaphor. Herbst 1974). S. 95-110.

Saiber, Arielle, und Henry S. Turner: Mathematics and the Imagination. A Brief Introduction. In: Configurations. A Journal of Literature, Science and Technology 17.1-2 (2009). S.1-18.

Shklovsky, Viktor: Art as Technique [1917]. Übers. von Lee T. Lemon und Marion J. Reis. In: Modern Criticism and Theory. Hrsg. von David Lodge. London und New York: Longman, 1988. S. 16-30. 
Vanderbeke, Dirk: Of Parts and Wholes: Self-similarity and Synecdoche in Science, Culture and Literature. In: Reconstruction 4.4 (Herbst 2004). http://reconstruction.eserver.org/Issues/ 044/vanderbeke.htm (14. April 2015).

Vanderbeke, Dirk: Theoretische Welten und literarische Transformationen. Tübingen: Niemeyer, 2004.

Weinberg, Steven: Dreams of a Final Theory. London: Vintage, 1993.

Weissert, Thomas P.: Representation and Bifurcation. Borges's Garden of Chaos Dynamics. In: Chaos and Order. Complex Dynamics in Literature and Science. Hrsg. von N. Katherine Hayles. Chicago und London: University of Chicago Press, 1991. S. 223-243.

Whewell, William: The Philosophy of the Inductive Sciences. Founded Upon Their History. Bd. 2. London: John W. Parker, 1840.

Wolf, Werner: Ästhetische Illusion und Illusionsdurchbrechung in der Erzählkunst. Tübingen: Niemeyer, 1993.

Whitehead, Alfred North: Prozess und Realität. Entwurf einer Kosmologie. [Process and Reality. An Essay in Cosmology, 1929]. Übers. von Hans Günther Holl. Frankfurt a. M.: Suhrkamp, 1987. 